\title{
La sieste comme indice de fragmentation du cycle de veille-sommeil : impact sur la cognition
}

Mathilde Reyt ${ }^{1^{*}}$, Alizée Latteur ${ }^{1^{*}}$, Christina Schmidt ${ }^{1,2}$, Vincenzo Muto ${ }^{1^{*}}$, Gregory Hammad $^{1 *}$

1. GIGA-CRC in Vivo Imaging, Université de Liège, Belgique

2. Psychology and Neurosciences of Cognition (PsyNCog), Université de Liège, Belgique

La fragmentation du cycle veille-sommeil augmente avec l'âge. Ici, nous explorons la sieste comme indicateur de cette fragmentation et son impact sur les performances cognitives.

Des données d'actimétrie ont été collectées chez 35 personnes âgées (57-85 ans, 19 siesteurs [sieste $>20 \mathrm{~min} /$ jour, $>3$ fois/semaine, depuis $>1$ an]). La fragmentation du sommeil a été évaluée grâce à la probabilité de transition d'une période de repos vers une période d'activité ( $k R A)$. Pour la fragmentation d'éveil, la probabilité de transition d'une période d'activité vers une période de repos (KAR) et la fraction de sommeil au cours de l'après-midi ( $\mathrm{fSoD}$ ) ont été examinées. Un score composite de cognition globale et un score spécifique des fonctions exécutives ont été calculés.

Une comparaison de groupes confirme que les siesteurs présentent une fragmentation de la période d'éveil plus élevée que les non siesteurs ( $\mathrm{fSoD}: \mathrm{p}<0.01$ ). Cependant, aucune différence n'est observée pour la fragmentation de sommeil ( $k R A: p=0.6)$. Une analyse multivariée (variables dépendantes: scores cognitifs, prédicteurs: indices d'actimétrie et variables démographiques) montre que le fSOD est associé à une diminution des performances cognitives $(p<0.05)$, plus particulièrement des fonctions exécutives $(p<0.01)$.

La fraction de sommeil au cours de l'après-midi mesurée par l'actimétrie peut être utilisée comme une mesure quantitative de la sieste. Cette mesure de fragmentation d'éveil semble jouer un rôle dans le déclin des performances cognitives au cours du vieillissement.

Financement: Belgian Fund for Scientific Research (FNRS), European Research Council (ERC-Starting Grant) 